\title{
Therapeutic Targets of Bufalin on Renal Carcinoma and Mechanisms: Experimental Validation of Network Pharmacology Analysis
}

\author{
Lei Zhang $\mathbb{D}^{1},{ }^{1}$ Yi-Ming Pan $\mathbb{D}^{1},{ }^{1}$ Lu-Yao Wang $\mathbb{D}^{1},{ }^{1}$ Wan-Zhu Zhao $\mathbb{D}^{\circ},{ }^{2}$ Shao-Cheng Yang $\mathbb{D}^{\circ},{ }^{1}$

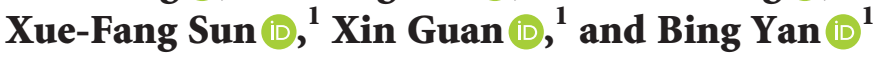 \\ ${ }^{1}$ College of Life Science and Technology, Mudanjiang Normal University, Mudanjiang 157011, China \\ ${ }^{2}$ School of Public Health, Xinjiang Medical University, Urumqi 830017, China \\ Correspondence should be addressed to Lei Zhang; swxzlz@126.com and Bing Yan; swxyb@126.com
}

Received 5 August 2021; Revised 15 November 2021; Accepted 24 November 2021; Published 28 January 2022

Academic Editor: Prashant Modi

Copyright (c) 2022 Lei Zhang et al. This is an open access article distributed under the Creative Commons Attribution License, which permits unrestricted use, distribution, and reproduction in any medium, provided the original work is properly cited.

\begin{abstract}
The possible targets underlying the activity of bufalin on renal cell carcinoma (RCC) were investigated using network pharmacology and experimental approaches. PharmMapper and other databases were explored for predicting the bufalin targets and RCC-related targets. Finally, the enriched pathways and the targets were analyzed by the Kyoto Encyclopedia of Genes and Genomes (KEGG) and Gene Ontology (GO) pathway enrichment analyses. Furthermore, in vitro cell experiments were used to verify bufalin activation of AKT and MAPK signaling pathways in human mesangial cells. The therapeutic targets related to bufalin were identified via 35 intersecting targets. GO analysis identified 29 molecular functions, 16 cellular components, and 91 biological processes. KEGG pathway annotation identified 15 signal transduction pathways and 4 tumor-related pathways.
\end{abstract}

\section{Introduction}

Bufalin $\left(\mathrm{C}_{24} \mathrm{H}_{34} \mathrm{O}_{4}\right)$, a major monomer component of toad venom, is extracted from toads for use in traditional Chinese medicine. It is derived from traditional Chinese medicine and includes a single component and is milder than Western medicines [1]. Bufalin has received increasing attention of researchers and clinicians due to its anticancer, heart-strengthening, analgesic, and other effects $[2,3]$. Bufalin has been shown to have antitumor efficacy against a variety of tumors, including mouth cancer, esophageal cancer, and bladder cancer [4-6]. Bufalin's anticancer action is distinguished by its low dose and highly selective nature. At the molecular level, studies employing bufalin have investigated the occurrence and growth of cancer cells, cell cycle, apoptosis, and gene expression. This study aimed to look into the biological signaling pathways and the predicted therapeutic targets of bufalin for treating RCC as well as providing experimental data and bioinformatics for basic clinical research on RCC treatment, using a scientific strategy based on network pharmacology.

\section{Materials and Methods}

2.1. The Target of Bufalin Resistance to RCC Was Obtained and Predicted. The PubChem website and PharmMapper server were used to predict potential targets for all the predicted targets of bufalin [7]. Additionally, the Draw Venn Diagram website (https://Bioinformatics.psb.ugent.be/webtools/Venn/ ) in the GeneCards database (https://www.Genecards.org/) [8] was used for intersection comparison, and the possible anti-RCC targets of bufalin were acquired from the GeneCards database (Figure 1).

2.2. PPI Network Construction and Topological Analysis of Bufalin. The anti-RCC protein-protein interaction (PPI) network was generated using the Cytoscape 3.7.2 software, and the target and target functional proteins were collected using the online String program (https://string-db.Org/). The maximum degree of freedom in the PPI network of topological parameters of the average sum was analyzed by using Cytoscape using the landscape analysis approach. 


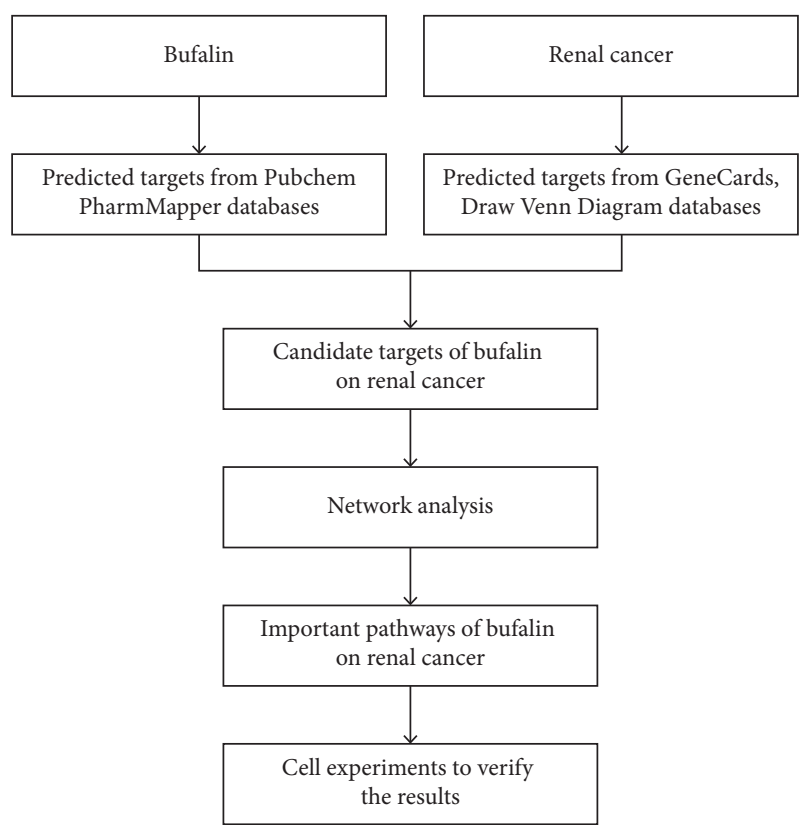

Figure 1: The analysis design of bufalin on renal carcinoma is depicted in a flowchart.

\subsection{GO Enrichment Analysis and KEGG Pathway Annotation.} The biological information annotation database (https:// David.NCIFCRF.gov/, David) [9] was used to conduct GO and KEGG analyses, and GO enrichment analysis covered biological processes, molecular function (MF), and cellular components (CC). The analysis results were downloaded, and the $P$ value $<0.05$ was set for further screening analysis and mapping.

\subsection{Replication Experiment}

2.4.1. Materials. Bufalin was purchased from Beijing Solaibao Technology Co. Ltd. (China). Caki-1 cells were donated by Harbin Institute of Technology cell laboratory, and human normal liver cells were retained by the Biology Department of Mudanjiang Normal University. All cell culture reagents were from HyClone (USA). The cell cycle detection kit and FITC kit were purchased from Invitrogen (Nanjing, China). PI3K and Akt1/2/3 were purchased from Santa (Wuhan, China). $\beta$-Actin was purchased from Boaosen (Wuhan, China).

2.4.2. Cytotoxicity Assay. Human normal liver cells were cultured in an incubator with $5 \%$ concentration of $\mathrm{CO}_{2}$, and the cells were inoculated into a 96-well culture plate at the rate of 1000 cells per well. The cytotoxic effects were assessed using the MTT assay. Experimental groups were treated with bufalin at the final concentration of $0.1,1$, and $10 \mu \mathrm{mol} / \mathrm{L}$ for 24 hours. All of the tests were carried out in triplicate, and the results were expressed as inhibition rate.

The cytotoxic effects were assessed using the MTT assay. Bufalin was added to the experimental group at final concentrations of $1,10,20,50,100$, and $1000 \mathrm{nmol} / \mathrm{L}$, while the conventional medium was added to the control group. The durations for the treatment were $12 \mathrm{~h}, 24 \mathrm{~h}$, and $48 \mathrm{~h}$. All of the tests were carried out in triplicate, and the results were expressed as the mean $\mathrm{IC}_{50}$ values.

2.5. Annexin V/PI Staining for Apoptotic Cell Death. In brief, bufalin was applied to Caki-1 cells for 12 and 24 hours at varying concentrations $(5,10,20,50,100$, and $200 \mathrm{nmol} / \mathrm{L})$ following the method reported by $\mathrm{Ni}$ et al. [10]. The cells were digested by using trypsin without EDTA and collected. The collected cell density was adjusted to $1 \times 10^{6}$ cells $/ \mathrm{mL}$ using $1 \mathrm{x}$ binding buffer. Annexin V-FITC $15 \mu \mathrm{L}$ and $5 \mu \mathrm{L}$ PI were added to cell suspension $(100 \mu \mathrm{L})$ and gently mixed. After staining, the cells were rinsed with PBS (containing $0.5 \%$ FBS). Finally, the cells were resuspended with $400 \mu \mathrm{L}$ binding buffer for flow cytometry analysis.

2.5.1. Cell Cycle Analysis. Various concentrations of bufalin $(1,5,10,20$, and $50 \mathrm{nmol} / \mathrm{L})$ were added to the cells for $24 \mathrm{~h}$, following the method reported by $\mathrm{Ni}$ et al. [11]. After culture, the sample was digested with $0.25 \%$ trypsin and $0.02 \%$ EDTA, and the cells were collected. The cells were cleaned with PBS twice and centrifuged at $4^{\circ} \mathrm{C}$ for $5 \mathrm{~min}$ at $1000 \mathrm{rpm}$, and the supernatant was discarded. The mixture was incubated at $37^{\circ} \mathrm{C}$ for $30 \mathrm{~min}$ at dark, which contains $10 \mu \mathrm{L}$ ribonuclease (RNaseA, $25 \mu \mathrm{g} / \mathrm{mL}$ ) and $10 \mu \mathrm{L}$ freshly prepared PI staining solution $(50 \mu \mathrm{g} / \mathrm{mL})$. Flow cytometry was used for analysis.

2.6. Western Blotting Analysis. Cells were treated with bufalin at concentrations of 10, 100, 500, and $1000 \mathrm{nmol} / \mathrm{L}$ and cultured for $24 \mathrm{~h}$, following the method reported by $\mathrm{Ni}$ et al. [11]. A total of $30 \mu \mathrm{g}$ protein from each well was separated by $8 \%$ (or 10\%) SDS-PAGE and transferred to polyvinylidene difluoride (PVDF) membranes. The membranes were blocked at room temperature for $1 \mathrm{~h}$ with $5 \%$ skim milk in tris-buffered saline with $0.1 \%$ Tween 20 (TBST), followed by incubation with the primary antibody diluted with $5 \%$ skim milk in TBST at $4^{\circ} \mathrm{C}$ overnight, and then incubated with the secondary antibody and detected using the ECL chemiluminescent system. Immune complexes were formed by incubation with the following antibodies: antiMAPK, anti-PI3K, and anti-AKT. Loading differences were normalized using a monoclonal $\beta$-actin antibody.

2.7. Statistical Analysis. The SPSS 13.0 statistical software was used for the data analysis. A $P$ value of less than 0.05 was considered statistically significant.

\section{Results}

3.1. Target Network Analysis. The top 100 target proteins were finally obtained in the PharmMapper server, and 73 target proteins with a $Z$-score $>0$ were selected, which were then imported into the UniProt database and corrected into gene symbol and gene ID. Furthermore, 30 goals were listed based on the $Z$-score ranking (Table 1 ). 
TABLE 1: Target prediction of bufalin.

\begin{tabular}{|c|c|c|c|c|c|c|c|}
\hline Pharma model & $Z$-score & Gene name & Gene ID & Pharma model & $Z$-score & Gene name & Gene ID \\
\hline $1 \mathrm{j} 78 \_\mathrm{v}$ & 2.92521 & GC & 2638 & 2is7_v & 1.00875 & AKR1B1 & 231 \\
\hline 1lho_v & 2.78452 & SHBG & 6462 & 1j96_v & 1.00818 & AKR1C2 & 1646 \\
\hline 2oi0_v & 2.69038 & Adam17 & 6868 & 2ywp_v & 0.980715 & CHEK1 & 1111 \\
\hline 1ov4_v & 2.31158 & SULT2A1 & 6822 & $2 \mathrm{p} 3 g \_v$ & 0.944171 & MAPKAPK2 & 9261 \\
\hline 1a28_v & 2.30115 & PGR & 5241 & $1 \mathrm{kbo} \_\mathrm{v}$ & 0.92543 & NQO1 & 1728 \\
\hline 2pix_v & 1.82985 & $\mathrm{AR}$ & 367 & 1ya3_v & 0.887381 & NR3C2 & 4306 \\
\hline 1xdd_v & 1.34837 & ITGAL & 3683 & 1gfw_v & 0.853259 & CASP3 & 836 \\
\hline $1 y d r \_v$ & 1.29681 & PRKACA & 282322 & 1csb_v & 0.828267 & CTSB & 1508 \\
\hline 1pmv_v & 1.25964 & MAPK10 & 5602 & 2h8h_v & 0.822228 & SRC & 6714 \\
\hline 2rku_v & 1.1589 & PLK1 & 5347 & 2zas_v & 0.819623 & ESRRG & 2104 \\
\hline $1 \mathrm{e} 7 \mathrm{e} \_v$ & 1.15408 & ALB & 213 & 1m9r_v & 0.817268 & NOS3 & 4846 \\
\hline 2qo8_v & 1.15252 & CA2 & 760 & 1n83_v & 0.80888 & RORA & 6095 \\
\hline 2oht_v & 1.14078 & BACE1 & 23621 & 2ocf_v & 0.693666 & ESR1 & 2099 \\
\hline 1p49_v & 1.08482 & STS & 412 & 1reu_v & 0.668496 & BMP2 & 650 \\
\hline $2 j 7 x \_v$ & 1.0527 & Esr2 & 25149 & 1161_v & 0.529143 & APOA2 & 336 \\
\hline
\end{tabular}

3.2. Bufalin-Related Targets for the Treatment of RCC. To obtain renal cancer-related disease targets, 1699 RCC-related targets were obtained using the GeneCards database. The intersection comparison between bufalin targets and RCC-related targets was conducted, and the Venn diagram identified 34 possible targets with connections to RCC and bufalin (Figure 2).

3.3. Bufalin-RCC Network. RCC and bufalin intersection targets were also added to the String database, and Cytoscape was utilized to create a functional protein-protein interaction network. The loop network contained 34 nodes that were linked and had 133 edges between them (Figure 3 ). The average local clustering coefficient was 0.714 , and the average node degree was 7.82 .

\subsection{Enrichment Analysis of the Bufalin-RCC Target Network}

3.4.1. GO Enrichment Analysis. The 34-target information was entered into the David database for GO enrichment analysis. The results showed that 91 items including signal transduction, cell proliferation, protein phosphorylation, and others were related to biological processes. Further, 29 items including enzyme binding and others were related to molecular function. Similarly, 16 items including the nucleus, cytoplasm, and mitochondria were related to cell components. Figure 4 and Table 2 show the top 10 BP, top 10 $\mathrm{CC}$, and top $10 \mathrm{MF}$.

3.4.2. Pathway of the Bufalin-RCC Network. The information of 34 targets was imported into the David database for the KEGG pathway study. Ratio and $P$ values were used to determine the degree of enrichment based on the number of target protein genes enriched in the pathway (Figure 5) [12]. After the analysis, 19 significant therapeutic pathways including epithelial cell signaling in Helicobacter pylori infection, proteoglycans in cancer, FoxO, GnRH, estrogen, pathways in cancer, Ras, prolactin, MAPK, TNF, Rap1, thyroid hormone, neurotrophin, sphingolipid, oxytocin,

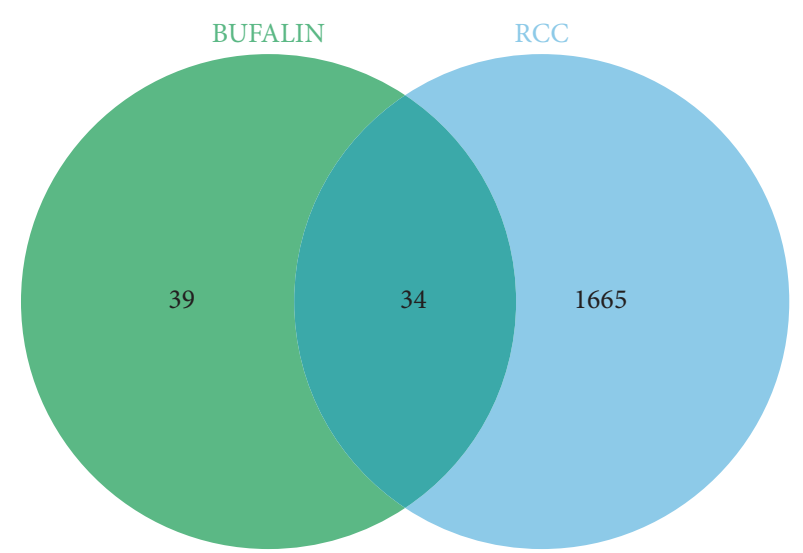

FIgURE 2: Venn diagram of bufalin targets and RCC-related pathogenesis targets.

VEGF, Fc epsilon RI, insulin resistance, and hepatitis C were identified. In addition, four pathways including cancer pathways, the FoxO signaling pathway, the MAPK signaling route, and proteoglycans in cancer had more than five targets.

3.5. The Inhibitory Effect of Bufalin on Cells. The inhibitory effect of bufalin on human normal liver cells was not obvious. The cells were treated with bufalin $(0.1,1$, and $10 \mu \mathrm{M})$ for 24 hours, and the inhibitory rate was $0.2 \pm 0.4 \%$, $2.6 \pm 0.6 \%$, and $20.7 \pm 0.5 \%$, respectively. Therefore, we selected bufalin at a lower concentration for the next experiment.

Low concentrations of bufalin had no inhibitory effect on a kidney carcinoma cell line (Caki-1); however, the effect of bufalin on the Caki-1 cells was dramatically amplified with increasing time and concentration. When observed under a microscope, the membrane of the renal cell carcinoma cells displayed a bubble shape when bufalin acted on the Caki-1 cells, and some cells gradually changed from spindle-shaped to round and lifted off the dish. 


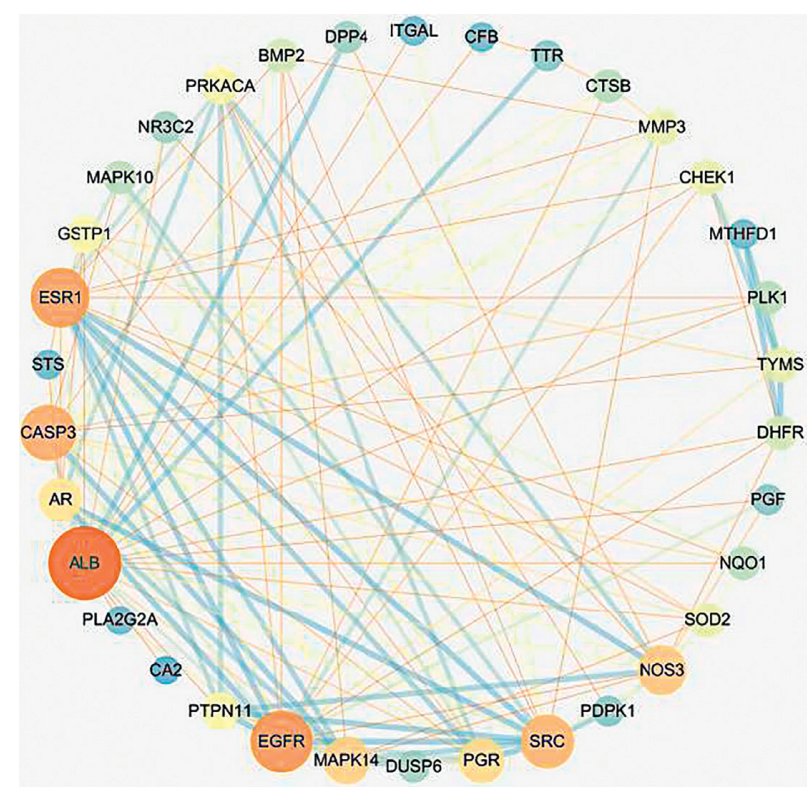

Figure 3: Protein interaction network of targets of bufalin on RCC. Blue represents the low degree, orange represents the high degree, and yellow represents the middle degree.

To investigate the anticancer effects of bufalin on Caki-1 cells, the cells were treated with bufalin $(0,1,10,20,50,100$, and $1000 \mathrm{nM}$ ) for 12,24 , or $48 \mathrm{~h}$. The MTT assay indicated that bufalin had a significant time and dose-dependent inhibitory effect on Caki- 1 cell proliferation. The $\mathrm{IC}_{50}$ values of bufalin on Caki-1 cells at 12, 24, and $48 \mathrm{~h}$ were $43.68 \pm 4.63,27.31 \pm 2.32$, and $18.06 \pm 3.46 \mathrm{nM}$, respectively (Figure 6). The OD values measured at 12, 24, and 48 hours after bufalin acts on cells and the inhibition rates of different concentrations of bufalin at different times are described in the Supplementary Materials (available here).

3.6. Bufalin Induces Apoptosis in Caki-1 Cells. The cells were treated with bufalin $(0,5,10,20,50,100$, and $200 \mathrm{nM})$ for 12 or 24 hours, and flow cytometry was performed after Annexin V-PI labeling to see if the bufalin-induced reduction in cell survival was due to apoptosis. As shown, the percentage of apoptotic cells at $12 \mathrm{~h}$ was $17.61 \%, 27.72 \%$, $29.71 \%, 29.97 \%, 40.71 \%, 46.36 \%$, and $50.97 \%$, respectively (Figures 7 and 8), and the values at $24 \mathrm{~h}$ were $17.61 \%, 27.41 \%$, $30.29 \%, 30.61 \%, 37.86 \%, 53.00 \%$, and $84.46 \%$, respectively (Figures 8 and 9). These findings showed that bufalin induced apoptosis and inhibited cell viability in gastric cancer cells.

3.7. Bufalin Induces Cell Cycle Arrest at the G2/M Phase. Flow cytometry was used to measure the number of Caki-1 cells in each phase to see if bufalin may cause cell cycle arrest. As evident from the results, bufalin increased the number of cells in the G2/M phase compared to the control treatment. The proportion of Caki-1 cells arrested in the G2/M phase increased sufficiently with increasing bufalin concentration, but the Caki-1 cell arrest proportion in the G2/M phase was significant in the $20 \mathrm{nmol} / \mathrm{L}$ and $50 \mathrm{nmol} / \mathrm{L}$ bufalin group.
We also observed an arrest in the S phase with $50 \mathrm{nmol} / \mathrm{L}$ bufalin. These results show that bufalin inhibited Caki-1 cell growth by causing cell cycle arrest in the G2/M phase (Figures 10 and 11).

The expression levels of proteins implicated in the MAPK and PI3K/Akt signaling pathways were evaluated when Caki-1 cells were exposed to bufalin $(10,100,500$, and $1000 \mathrm{nM}$ ) for 24 hours to study the signal transduction route through which bufalin influences tumor growth. We observed that the level of MAPK and PI3K was decreased after $24 \mathrm{~h}$ in a dose-dependent manner, leading to a decrease in its downstream molecule p-Akt (Figure 12).

\section{Discussion}

Toxicity and resistance to currently available chemotherapeutic drugs are important barriers to cancer therapy. As a result, the ideal method would be to search for new and effective anticancer drugs with fewer side effects [13]. Bufalin is the main ingredient of the toad venom, causing cell death by inducing cell apoptosis and blocking the cell cycle in human cancer cells $[14,15]$. In human liver cancer, prostate cancer, and multiple myeloma, bufalin has been shown to induce apoptosis and decrease proliferation [16-18]. However, it causes apoptosis and suppresses proliferation in Caki-1 cells via a molecular mechanism that is yet to be discovered. The study screened and discovered bufalin's therapeutic targets in RCC as well as the biological mechanisms that underpin them.

The experiments showed that bufalin exerted an antiproliferative effect on Caki-1 cells in a concentration-dependent manner. The cells underwent morphological changes, including reduced cell proliferation, circular shrinkage, and reduced adherence, which may be caused by the destruction of the cell membrane, cytoskeleton, and 


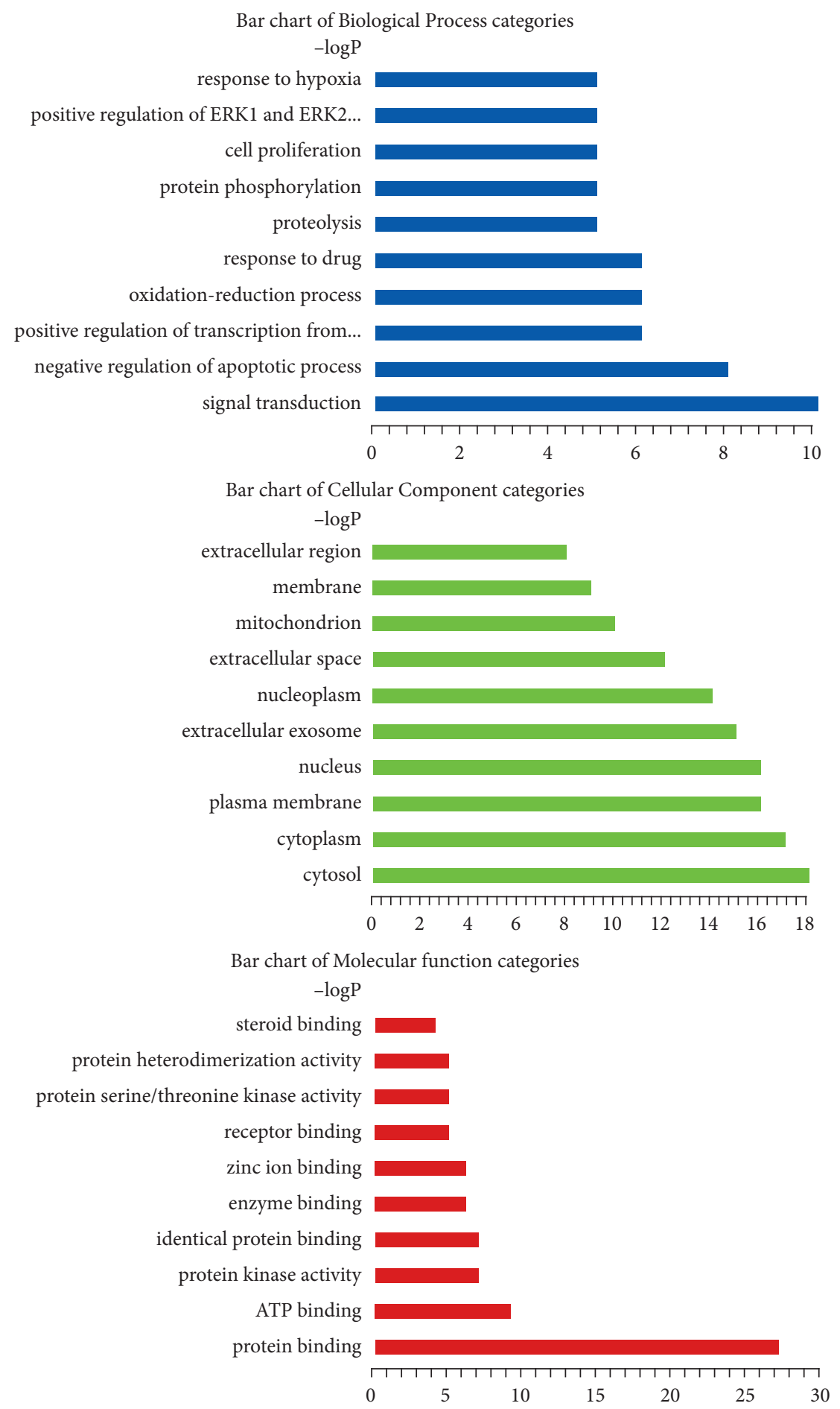

FIGURE 4: GO enrichment plot of bufalin resistance to RCC targets.

some internal cell structures by bufalin, resulting in significant morphological changes.

After treating Caki-1 cells with bufalin for 24 hours, the fraction of cells in the G0/G1 phase of the cell cycle was reduced significantly, while the fraction of cells in the G2/M phase continued to increase which is essentially consistent with the findings of Suehiro et al. and Ni et al. [10, 19]. This study also revealed that the number of cells in the G2/M phase increased as the bufalin concentration increased which is extremely significant, and when cycling cells face stress, they are arrested at one of the two cell cycle checkpoints, G2/M or G1/S.

Here, a "compound-target-pathway-disease" network was built using the network pharmacology approach to identify possible bufalin anti-RCC targets. Through KEGG pathway annotation and GO enrichment analysis, the target 
TABle 2: The findings of the GO analysis, which comprised the top $10 \mathrm{MF}$, top $10 \mathrm{CC}$, and top $10 \mathrm{BP}$.

\begin{tabular}{|c|c|c|}
\hline GO term & Gene ratio & Ontology \\
\hline Signal transduction & 10 & $\mathrm{BP}$ \\
\hline Negative regulation of the apoptotic process & 8 & $\mathrm{BP}$ \\
\hline Positive regulation of transcription from RNA polymerase II promoter & 6 & $\mathrm{BP}$ \\
\hline Oxidation-reduction process & 6 & $\mathrm{BP}$ \\
\hline Response to drug & 6 & $\mathrm{BP}$ \\
\hline Proteolysis & 5 & $\mathrm{BP}$ \\
\hline Protein phosphorylation & 5 & $\mathrm{BP}$ \\
\hline Cell proliferation & 5 & $\mathrm{BP}$ \\
\hline Positive regulation of ERK1 and ERK2 cascade & 5 & $\mathrm{BP}$ \\
\hline Response to hypoxia & 5 & $\mathrm{BP}$ \\
\hline Cytosol & 18 & $\mathrm{CC}$ \\
\hline Cytoplasm & 17 & $\mathrm{CC}$ \\
\hline Plasma membrane & 16 & $\mathrm{CC}$ \\
\hline Nucleus & 16 & $\mathrm{CC}$ \\
\hline Extracellular exosome & 15 & $\mathrm{CC}$ \\
\hline Nucleoplasm & 14 & $\mathrm{CC}$ \\
\hline Extracellular space & 12 & CC \\
\hline Mitochondrion & 10 & $\mathrm{CC}$ \\
\hline Membrane & 9 & $\mathrm{CC}$ \\
\hline Extracellular region & 8 & $\mathrm{CC}$ \\
\hline Protein binding & 27 & MF \\
\hline ATP binding & 9 & MF \\
\hline Protein kinase activity & 7 & MF \\
\hline Identical protein binding & 7 & $\mathrm{MF}$ \\
\hline Enzyme binding & 6 & MF \\
\hline Zinc ion binding & 6 & $\mathrm{MF}$ \\
\hline Receptor binding & 5 & $\mathrm{MF}$ \\
\hline Protein serine/threonine kinase activity & 5 & $\mathrm{MF}$ \\
\hline Protein heterodimerization activity & 5 & $\mathrm{MF}$ \\
\hline Steroid binding & 4 & MF \\
\hline
\end{tabular}

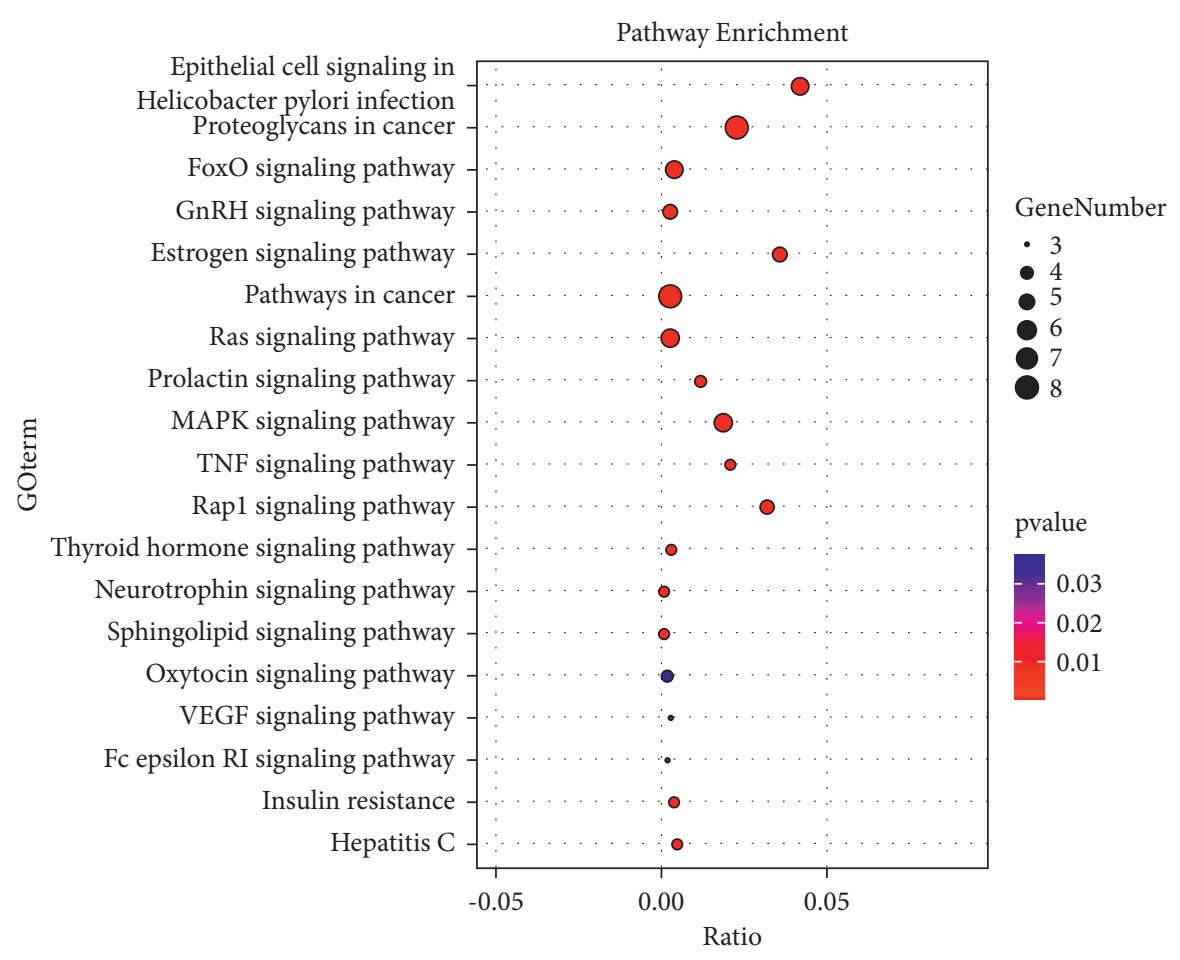

FIGURE 5: KEGG pathway enrichment diagram. 


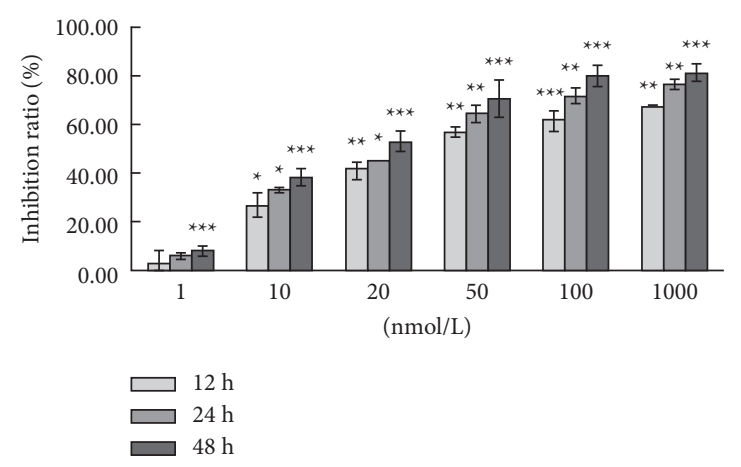

Figure 6: Bufalin inhibits the proliferation of Caki-1 cells.

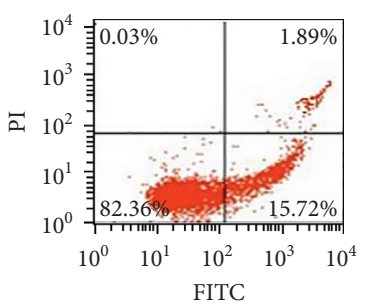

(a)

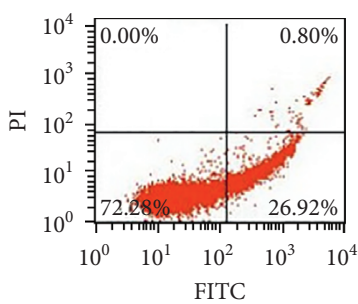

(b)

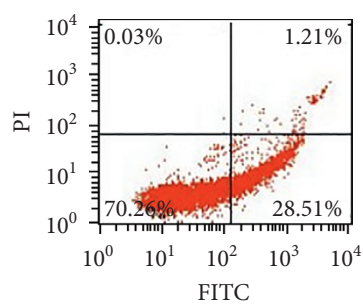

(c)

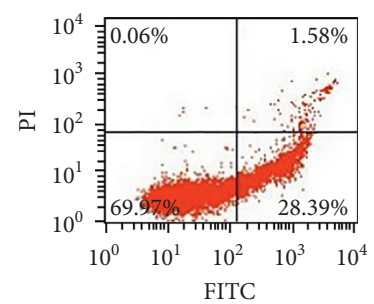

(d)

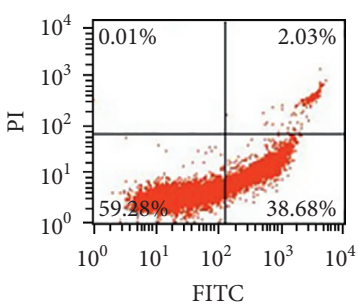

(e)

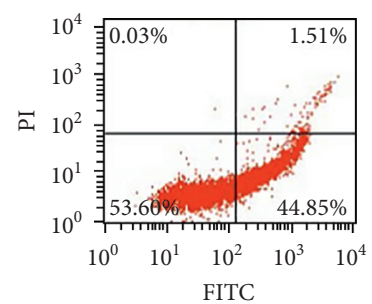

(f)

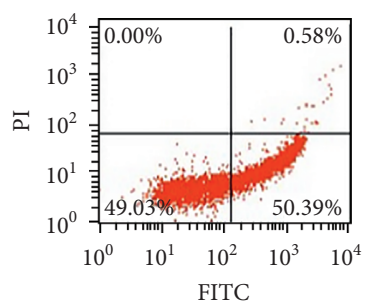

(g)

Figure 7: Bufalin induces apoptosis in Caki-1 cells (12 h): (a) Control; (b) $5 \mathrm{nmol} / \mathrm{L} ;$ (c) $10 \mathrm{nmol} / \mathrm{L}$; (d) $20 \mathrm{nmol} / \mathrm{L}$; (e) $50 \mathrm{nmol} / \mathrm{L}$; (f) $100 \mathrm{nmol} / \mathrm{L} ;(\mathrm{g}) 200 \mathrm{nmol} / \mathrm{L}$.

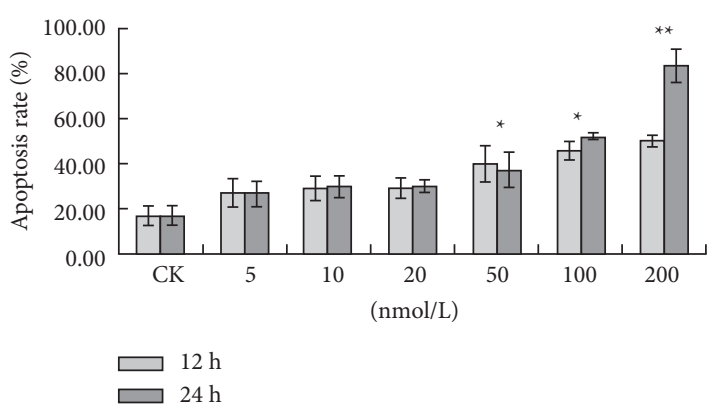

FIgure 8: Bufalin induces apoptosis in Caki-1 cells (12 h and $24 \mathrm{~h}$ ): (a) Control; (b) $5 \mathrm{nmol} / \mathrm{L}$; (c) $10 \mathrm{nmol} / \mathrm{L}$; (d) $20 \mathrm{nmol} / \mathrm{L}$; (e) $50 \mathrm{nmol} / \mathrm{L}$; (f) $100 \mathrm{nmol} / \mathrm{L}$; (g) $200 \mathrm{nmol} / \mathrm{L}$.

protein genes of bufalin contain anti-RCC including MAPK10 and PI3K. These target protein genes are involved in multiple tumor pathways and signal transduction pathways $[20,21]$. In this study, the predicted potential targets were annotated through the KEGG pathway to obtain signal transduction pathways related to RCC, such as MAPK and
PI3K signaling pathway. These pathways are linked to the development and occurrence of tumors.

One of the most significant routes in the eukaryotic signal transmission network is the mitogen-activated protein kinase (MAPK) chain, which is involved in gene expression control and cytoplasmic functional activities 


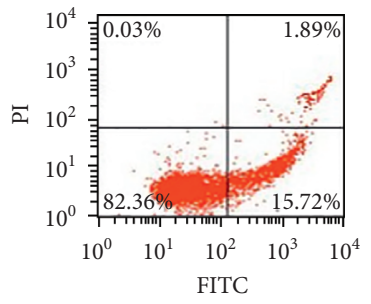

(a)

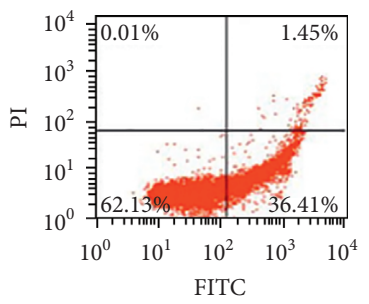

(e)

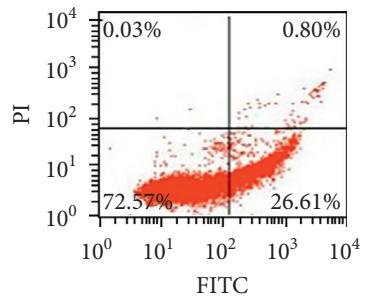

(b)

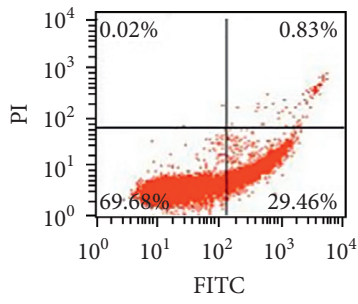

(c)

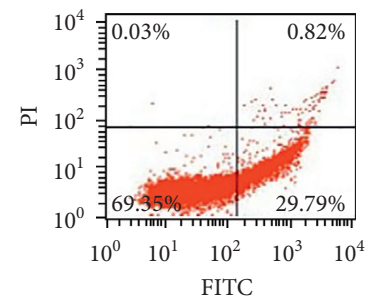

(d)

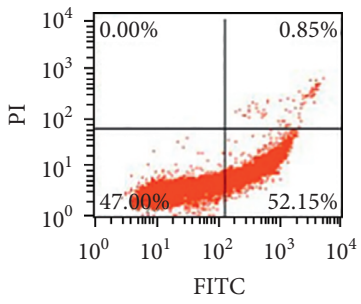

(f)

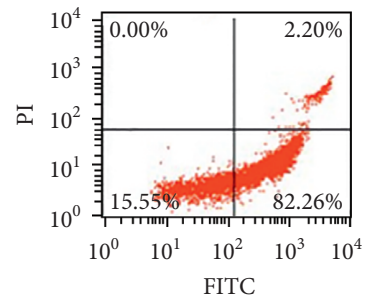

(g)

Figure 9: Bufalin induces apoptosis in Caki-1 cells (24h): (a) Control; (b) $5 \mathrm{nmol} / \mathrm{L}$; (c) $10 \mathrm{nmol} / \mathrm{L}$; (d) $20 \mathrm{nmol} / \mathrm{L}$; (e) $50 \mathrm{nmol} / \mathrm{L}$; (f) $100 \mathrm{nmol} / \mathrm{L}$; (g) $200 \mathrm{nmol} / \mathrm{L}$.

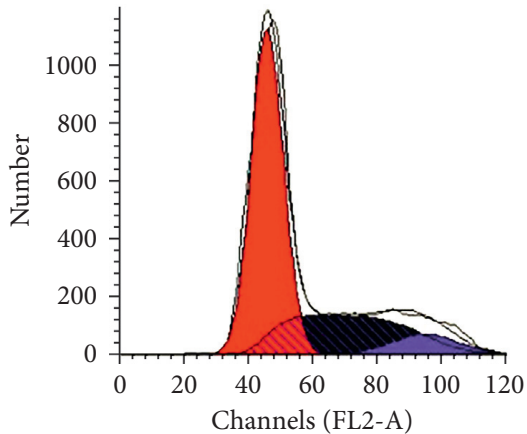

$\square$ Apoptosis

$$
\begin{aligned}
& \text { Dip G1 } \\
& \text { Dip G2 } \\
& \text { Dip S }
\end{aligned}
$$

(a)

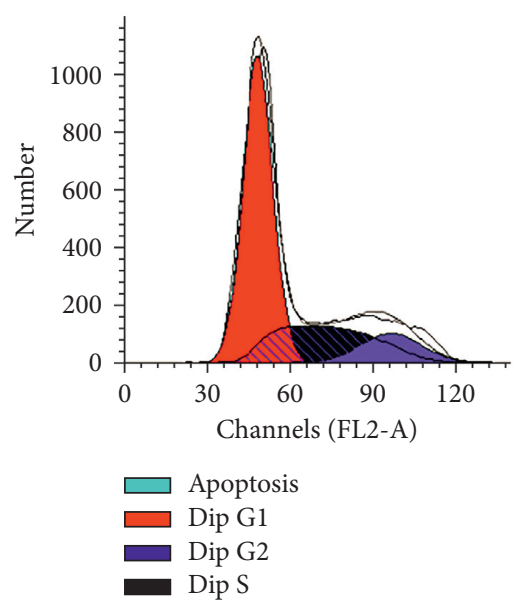

(d)

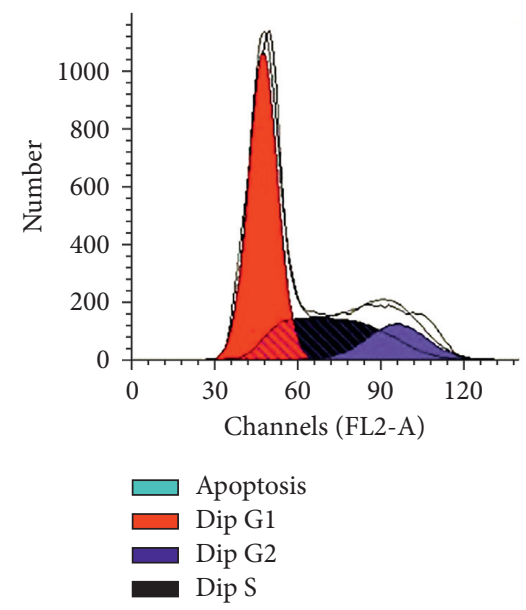

(b)

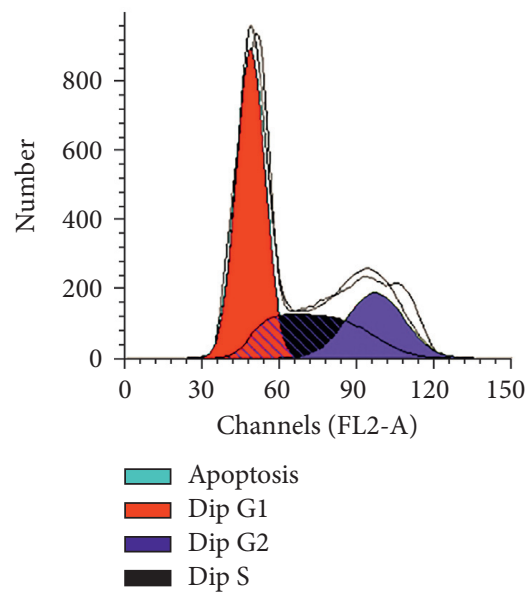

(e)

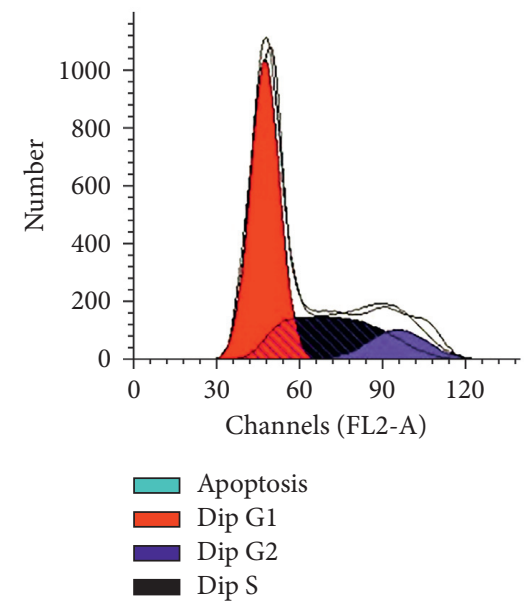

(c)

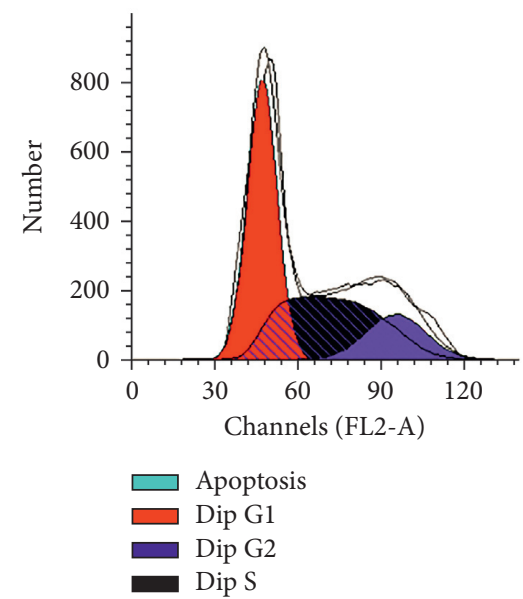

(f)

Figure 10: Bufalin induces cell cycle arrest in RCC Caki-1: (a) CK; (b) $1 \mathrm{nmol} / \mathrm{L}$; (c) $5 \mathrm{nmol} / \mathrm{L}$; (d) $10 \mathrm{nmol} / \mathrm{L}$; (e) $20 \mathrm{nmol} / \mathrm{L}$; (f) $50 \mathrm{nmol} / \mathrm{L}$. 


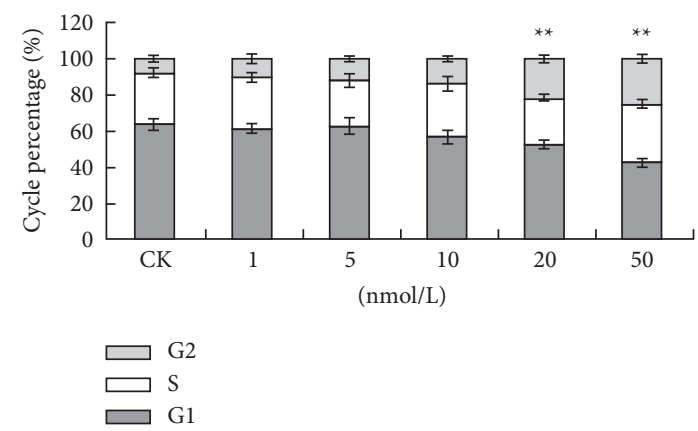

Figure 11: Bufalin induces cell cycle arrest in RCC Caki-1: (a) CK; (b) $1 \mathrm{nmol} / \mathrm{L}$; (c) $5 \mathrm{nmol} / \mathrm{L}$; (d) $10 \mathrm{nmol} / \mathrm{L}$; (e) $20 \mathrm{nmol} / \mathrm{L}$; (f) $50 \mathrm{nmol} / \mathrm{L}$.

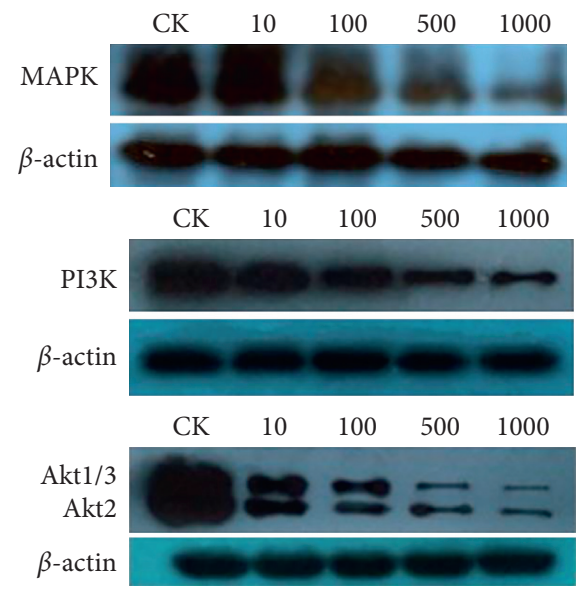

Figure 12: The expression of MAPK, P13K, and Akt protein after bufalin.

[22]. The MAPK pathway is one of the common intersections of signal transduction pathways, such as stress, inflammation, cell proliferation, differentiation, functional synchronization, transformation, and apoptosis, and the extracellular signal by the receptor, $\mathrm{G} / \mathrm{G}$ of protein, protein kinase, and transcription factors, such as signal network, passed to the intracellular, involved in cell proliferation, differentiation, cancerous, transfer, and apoptosis [23]. In this study, we found that the levels of PI3K and MAPK were reduced after 24 hours in a dose-dependent manner. Therefore, we suggest that bufalin may inhibit the proliferation of Caki-1 cells by inhibiting the MAPK and PIK signaling pathways. These findings support bufalin's clinical use as an anticancer drug due to its therapeutic effects in renal cell carcinoma. However, we believe that more animal experiments are needed to better evaluate bufalin's therapeutic potential.

\section{Conclusion}

The possible targets of the bufalin on renal cell carcinoma were investigated using network pharmacological and experimental approaches. The bufalin-related therapeutic targets were identified via 35 intersecting targets. GO analysis identified 29 molecular functions, 16 cellular components, and 91 biological processes. KEGG pathway annotation identified 15 signal transduction pathways and 4 tumor-related pathways.

\section{Data Availability}

The data used to support the findings of this study are included with the Supplementary Information files.

\section{Conflicts of Interest}

The authors declare that they have no conflicts of interest regarding the publication of this paper.

\section{Acknowledgments}

This work was supported by the previous research project of the Heilongjiang Postdoctoral Talent Program (grant no. LBH-Q19190) and basic scientific research operating expenses of Provincial Universities in Heilongjiang Province (grant nos. QN2021003, 1355ZD006, 1354MSYTD007, and 202110233034).

\section{Supplementary Materials}

The number of Caki-1 cells was significantly changed after treatment with bufalin. Results indicated that bufalin can inhibit the proliferation of Caki-1 cells with a time- and dose-dependent manner (Table 1 and Figure 1). The OD values measured at 12,24, and 48 hours after bufalin acts on cells and the inhibition rates of different concentrations of bufalin at different times are described (Table 2). () (Supplementary Materials)

\section{References}

[1] Y.-G. Fang, P.-F. Wang, H.-D. Zhu et al., "Study on quality standard of Bufonis Venenum and its processed slice, toad venom powder," Zhongguo Zhongyao Zazhi=China Journal of Chinese Materia Medica, vol. 45, pp. 1726-1733, 2020.

[2] L. L. Wen, X. B. Xie, W. Huang et al., "Bufalin's anti-inflammatory and analgesic effects on NF-B signaling pathway," Journal of Sun Yat-Sen University(Medical Science Edition), vol. 35, no. 05, pp. 680-684+667, 2014.

[3] W.-W. Huang, J.-S. Yang, S.-J. Pai et al., "Bufalin induces G0/ G1 phase arrest through inhibiting the levels of cyclin D, cyclin E, CDK2 and CDK4, and triggers apoptosis via mitochondrial signaling pathway in T24 human bladder cancer cells," Mutation Research: Fundamental and Molecular Mechanisms of Mutagenesis, vol. 732, no. 1-2, pp. 26-33, 2012.

[4] S. Shen, Y. Zhang, Z. Wang, R. Zhang, and X. Gong, "Bufalin induces the interplay between apoptosis and autophagy in glioma cells through endoplasmic reticulum stress," International Journal of Biological Sciences, vol. 10, no. 2, pp. 212-224, 2014.

[5] Y. Ding, W. Liu, X. Wang et al., "Bufalin induces apoptosis in human esophageal carcinoma ECA109 cells by inhibiting the activation of the mTOR/p70S6K pathway," Oncology Letters, vol. 15, no. 6, pp. 9339-9346, 2018.

[6] H.-Y. Chou, F.-S. Chueh, Y.-S. Ma et al., "Bufalin induced apoptosis in SCC-4 human tongue cancer cells by decreasing 
Bcl-2 and increasing Bax expression via the mitochondriadependent pathway," Molecular Medicine Reports, vol. 16, no. 6, pp. 7959-7966, 2017.

[7] S. F. Zhao and X. J. Zhang, "Anticancer mechanism of ailanthone via reverse molecular docking and network pharmacological technology," Chinese Traditional and Herbal Drugs, vol. 49, pp. 4085-4092, 2018.

[8] H. Li and H. Liu, "Investigating the mechanisms of action of depside salt from salvia miltiorrhiza using bioinformatic analysis," Evidence Based Complement Alternative Medicine, vol. 2017, Article ID 5671860, 8 pages, 2017.

[9] L. Zeng, K. Yang, H. Liu, and G. Zhang, "A network pharmacology approach to investigate the pharmacological effects of Guizhi Fuling Wan on uterine fibroids," Experimental and Therapeutic Medicine, vol. 14, no. 5, pp. 4697-4710, 2017.

[10] T. Ni, H. Wang, D. Li et al., "Huachansu Capsule inhibits the proliferation of human gastric cancer cells via Akt/mTOR pathway," Biomedicine \& Pharmacotherapy, vol. 118, Article ID 109241, 2019.

[11] J. Xie, W. Lin, L. Huang et al., "Bufalin suppresses the proliferation and metastasis of renal cell carcinoma by inhibiting the PI3K/Akt/mTOR signaling pathway," Oncology Letters, vol. 16, no. 3, pp. 3867-3873, 2018.

[12] S. Yang, C.-C. Duan, R.-Y. Yan, and J.-Y. Zhang, "Activecomponent and integrative mechanism of Scutellaria barbatain treatment of cancer based on network pharmacology method," Chinese Traditional and Herbal Drugs, vol. 49, no. 15, pp. 3471-3482, 2018.

[13] E. Y. Su, Y. L. Chu, F. S. Chueh et al., "Bufalin induces apoptotic cell death in human nasopharyngeal carcinoma cells through mitochondrial ROS and TRAIL pathways," The American Journal of Chinese Medicine, vol. 47, no. 1, pp. 237-257, 2019.

[14] C. Zhang, K. Ma, and W.-Y. Li, "Cinobufagin suppresses the characteristics of osteosarcoma cancer cells by inhibiting the IL-6-OPN-STAT3 pathway," Drug Design, Development and Therapy, vol. 13, pp. 4075-4090, 2019.

[15] H.-Y. Qi, X.-J. Qu, J. Liu et al., "Bufalin induces protective autophagy by Cbl-b regulating mTOR and ERK signaling pathways in gastric cancer cells," Cell Biology International, vol. 43, no. 1, pp. 33-43, 2019.

[16] F. Qi, A. Li, Y. Inagaki et al., "Antitumor activity of extracts and compounds from the skin of the toad Bufo bufo gargarizans Cantor," International Immunopharmacology, vol. 11, no. 3, pp. 342-349, 2011.

[17] Y.-Q. Dong, W.-L. Ma, J. Gu, and W.-L. Zheng, "Effect of cinobufagin on nuclear factor-kappaB pathway in HepG2 cells," Nan Fang Yi Ke Da Xue Xue Bao= Journal of Southern Medical University, vol. 30, pp. 137-139, 2010.

[18] C.-H. Yu, S.-F. Kan, H.-F. Pu, E. Jea Chien, and P. S. Wang, "Apoptotic signaling in bufalin- and cinobufagin-treated androgen-dependent and -independent human prostate cancer cells," Cancer Science, vol. 99, no. 12, pp. 2467-2476, 2008.

[19] Y. Suehiro, Y. Takemoto, A. Nishimoto et al., "Dclk1 inhibition cancels 5-FU-induced cell-cycle arrest and decreases cell survival in colorectal cancer," Anticancer Research, vol. 38, no. 11, pp. 6225-6230, 2018.

[20] X.-F. Xu, F. Liu, J.-Q. Xin et al., "Respective roles of the mitogen-activated protein kinase (MAPK) family members in pancreatic stellate cell activation induced by transforming growth factor- $\beta 1$ (TGF- $\beta 1$ )," Biochemical and Biophysical Research Communications, vol. 501, no. 2, pp. 365-373, 2018.
[21] W. Liu and J. Li, “Theaflavin-3, 3'-digallate attenuates rheumatoid inflammation in mice through the nuclear factor$\kappa \mathrm{B}$ and MAPK pathways," Archivum Immunologiae et Therapiae Experimentalis, vol. 67, no. 3, pp. 153-160, 2019.

[22] E. K. Kim and E.-J. Choi, "Compromised MAPK signaling in human diseases: an update," Archives of Toxicology, vol. 89, no. 6, pp. 867-882, 2015.

[23] L. A. M. D. Santos, G. B. Rodrigues, F. V. B. Mota et al., "New thiazolidinedione LPSF/GQ-2 inhibits $\mathrm{NF} \kappa \mathrm{B}$ and MAPK activation in LPS-induced acute lung inflammation," International Immunopharmacology, vol. 57, pp. 91-101, 2018.

[24] D. Szklarczyk, A. Franceschini, S. Wyder et al., "STRING v10: protein-protein interaction networks, integrated over the tree of life," Nucleic Acids Research, vol. 43, no. D1, pp. D447-D452, 2015. 